


\title{
Estilos de vida, nivel de actividad física y prevalencia de obesidad de la población estudiantil universitaria
}

\author{
College students' lifestyles, physical activity level and obesity prevalence threatens to develop \\ diseases affecting their quality life \\ Tyrone Mauricio Loría Calderón ${ }^{1}$ \\ Recibido: 29 /7/ 2016 / Aprobado: 1/11/2016
}

\begin{abstract}
Resumen
La conducta sedentaria es una amenaza importante para la salud y calidad de vida, aumentando el factor de riesgo de desarrollo de enfermedades no transmisibles y la muerte prematura. Es importante para comprender los patrones sedentarios y su relación con otros factores de riesgo, como la obesidad; este artículo busca describir el estilo de vida, el nivel de sedentarismo, la actividad física y la prevalencia de la obesidad en los estudiantes de la Universidad de Costa Rica (UCR), Recinto de Tacares. Ciento veintiún estudiantes informaron de su actividad física regular dentro de una semana normal de clases y una semana de descanso académico. Los resultados de este estudio muestran que los estudiantes pasaron la mayor parte de su tiempo en la conducta sedentaria y el comportamiento sedentario también está significativamente relacionado con un IMC superior.
\end{abstract}

Palabras clave: Actividad física, estilos de vida, obesidad, universitario.

\section{Abstract}

\begin{abstract}
A sedentary behavior is an important threat for students' health and their quality of life. It increases the risk to develop non-transferable diseases and a premature death. This study shows a description of the lifestyles, sedentary level, and obesity prevalence of students registered in Universidad de Costa Rica (UCR), Recinto de Tacares. The data is based on information gathered from one hundred twenty one undergraduates that describe their daily physical activity in a regular week of classes and an academic rest week. The results show that these college students spend most of the time in a sedentary behavior which it is significantly related to a superior IMC.
\end{abstract}

Key words: Physical activity, lifestyles, obesity, university.

\section{Introducción}

Los tiempos están cambiando y con ello las formas de enfrentar los escenarios que la vida de cada individuo; por tanto el estudio se orienta a brindar un aporte al mejoramiento de la calidad de vida de los estudiantes, se considera necesario conocer cuál es la situación con respecto a los estilos de vida, el nivel de actividad física realizado y el grado de obesidad en esta población. Esto con el fin de poder tomar acciones que repercutan en la vida de cada estudiante universitario y se mantenga a lo largo de sus vidas y lo puedan transmitir a otros.
Al buscar las líneas de investigación se ha encontrado que los estilo de vida en salud comprenden patrones de conducta relacionados con la salud, valores y actitudes adoptados por los individuos y los grupos en respuesta a su ambiente social y económico (Abel, 1991, citado por Oblitas, 2006). La actividad física es el movimiento corporal producido por la contracción de los músculos esqueléticos, el cual incrementa sustancialmente el gasto energético en reposo (ACSM, 2010). Además, la obesidad es una acumulación anormal o excesiva

\footnotetext{
${ }^{1}$ Magister, profesor e investigador del Departamento de Educación, Sede de Occidente, Universidad de Costa Rica. Correo electrónico: tloria13@gmail.com
} 
de grasa que puede ser perjudicial para la salud. Una forma simple de medir la obesidad es el índice de masa corporal (IMC) (OMS, 2011).

El término "estilo de vida" fue difundido y consolidado por Alvin Toffler en 1939, como se cita en Osorio (2002) en un momento de la historia donde cambia la sociedad, de ser homogénea en su actuar a una sociedad heterogénea. En épocas más recientes los estilos de vida se definen por Tavera, en Sanabria, et al. (2007), como los tipos de hábitos, actitudes, conductas, tradiciones, actividades y decisiones de una persona o de cierto grupo de personas, frente a las diversas circunstancias en las que el ser humano se desarrolla en sociedad, o mediante su quehacer diario y son susceptibles de ser modificadas.

Para la década del 2000, los estilos de vida saludable son una estrategia global, que hace parte de una tendencia moderna de salud; básicamente está enmarcada dentro de la prevención de enfermedades y promoción de la salud, tal vez el momento clave de esta tendencia fue en el año 2004 y se generó por una declaración de la Organización Mundial de Salud (OMS) para mejorar los factores de riesgo, como la alimentación poco saludable y el sedentarismo (Seignón, 2008).

Resulta importante trabajar bajo los mismos criterios que plantea la OMS para hacer cambios significativos a nivel de la población universitaria, en este caso con muy poco estímulo en el tema de estilos de vida saludable. La actividad física tiene un papel importante en este estudio, como mencionan Gómez, et al. (2005), en la actualidad nadie duda que la actividad física y el ejercicio, realizados de forma moderada y médicamente controlados, inciden de forma positiva sobre la salud y el estado de bienestar general de las personas, ya que un hábito de vida físicamente activo conlleva estilos de vida saludables, alejados del consumo de tóxicos y de los hábitos insanos.

Cabe señalar que la inactividad física no solo contribuye a las enfermedades crónicas no transmisibles (ECNT), sino que también puede predisponer a enfermedades mentales, la acumulación de estrés, un menor rendimiento escolar e incluso tener un efecto negativo en la interacción social (OPS, 2006). Para el Colegio Americano de Medicina del Deporte, una persona sedentaria es aquella que no realiza actividades físicas durante 30 minutos al día, al menos tres veces a la semana. El ente ha definido recomendaciones en cuanto a la regularidad, frecuencia y duración de la actividad; se debe de realizar entre 20-60 minutos continuos o intermitentes, con un mínimo de 3 veces a la semana y a una intensidad del 55-65\% hasta el 90\% de la frecuencia cardiaca (Scott, et al. 2000). El no cumplir con estas recomendaciones mínimas de actividad física, así como una mala nutrición (factores negativos en los estilos de vida saludable) pueden ocasionar altos porcentajes de grasa y caer en la obesidad. Actualmente, la obesidad se ha convertido en un problema de salud pública que no solo afecta a la situación estética, si no que se trata de una enfermedad crónica, multifactorial y muy complicada de tratar.

El diario de la Asociación Médica Americana (JAMA, por sus siglas en inglés) explica que los individuos con sobrepeso u obesos tienen mayores probabilidades de presentar problemas de salud que los que mantienen un peso saludable. Consumir mayor número de calorías (una medida de la energía proporcionada por los alimentos o líquidos) que las que necesita el cuerpo provoca un incremento de peso. Factores frecuentes que originan obesidad son estilos de vida basados en raciones demasiado generosas, fácil acceso a alimentos con escaso valor nutritivo y sedentarismo (JAMA, 2003).

Con base en lo anterior, se planteó este estudio para determinar las actividades que realizaban los estudiantes universitarios diariamente, el nivel de actividad física que tenían y la prevalencia de obesidad de estos. 


\section{Metodología}

\section{Tipo de investigación}

Esta investigación se plantea como un estudio transversal, de tipo descriptivo y exploratorio. El proyecto de investigación se limita a una sola observación en el tiempo y no se manipulan de variables.

\section{Sujetos y su selección}

Se trabajó con población estudiantil universitaria del Recinto de Grecia, Universidad de Costa Rica (UCR, Recinto de Grecia). Un total de 121 (53 hombres y 68 mujeres) estudiantes (19.79 \pm 2.46 años) fueron escogidos aleatoriamente de un total de 789 estudiantes del total del recinto y de trece diferentes carreras, la investigación fue realizada en el primer semestre de 2013. La muestra fue escogida de forma aleatoria simple. El 88.41\% de los estudiantes solo estudian y $11.59 \%$ trabajan fin de semana o entre semana o ambos. Cada estudiante llenó el "Consentimiento Informado" de la Institución.

\section{Instrumentos}

Para caracterizar los estilos de vida se aplicó la "Encuesta Hábitos de los Jóvenes" elaborada por Gil, et al. (2011), con algunas modificaciones en el encabezado y en el vocabulario de acuerdo con el caso en estudio y la población de referencia. Se modificaron algunas palabras tales como: "ordenador" por "computadora" y "cañas" por "cervezas", en la pregunta 13 la expresión "botellón” por la frase "te reúnes con tus amigos a consumir bebidas alcohólicas en zonas públicas".

La variable "actividad física" se midió con el cuestionario QAPACE elaborado por Nicolás Barbosa, y otros en el 2007, este cuestionario ha demostrado su fiabilidad y precisión para estudios epidemiológicos y la posibilidad de ser utilizado en estudios de población general; su reproductibilidad es de CCI $=0,96$ y su validez de $r=0,63$ (Barbosa y otros, 2007). QAPACE está diseñado para trabajar con estudiantes, posee, como una de sus características relevantes, el hecho de que permite establecer la diferencia entre gasto energético diario durante periodo de clases y periodo de vacaciones. Este cuestionario permite efectuar el cálculo del gasto energético diario a partir del auto reporte de actividades diarias en doce categorías: sueño, aseo, comidas, transporte, clases, actividad física obligatoria, otras actividades en el centro educativo, actividades fuera del centro educativo, actividades en vacaciones, actividades artísticas personales, deportes de competición y actividades caseras. En el QAPACE, se modificaron aspectos de forma y contenido.

El nivel de actividad física se obtuvo con la fórmula $\mathrm{PAL}=\mathrm{TEE} / \mathrm{BMR}$, propuesta por la Organización de las Naciones Unidas para la Agricultura y la Alimentación, la Organización Mundial de la Salud y la Universidad de las Naciones Unidas (FAO/WHO/UNU) (FAO, 2004), al ser $\mathrm{PAL}=$ nivel de actividad física, $\mathrm{TEE}=$ gasto energético total y $\mathrm{BMR}=$ tasa metabólica basal. El gasto energético total se obtiene a partir del cuestionario QAPACE y la tasa metabólica basal se estima con la fórmula de Schofield (FAO/WHO/ UNU) (FAO, 2004), la cual toma en cuenta la edad, género y peso de los individuos (este dato se dio automáticamente desde la romana empleada para el peso (Tanita BC-553), que usa esta misma fórmula y su validez y confiabilidad se encuentra en 2002 Nutrition Week: A Scientific and Clinical Forum and Exposition Title: International Comparison: Resting Energy Expenditure Prediction Models:The Americam Journal of Clinical Nutrition).

Para obtener el Índice de Masa Corporal (IMC) se dio mediante la fórmula para IMC=Peso $(\mathrm{Kg}) / \mathrm{Talla}^{2}(\mathrm{~m})$. Se tomó el peso en kilogramos con una báscula (marca y modelo Tanita Bc-553) y la talla en metros con un tallímetro portátil.

Por otra parte, se aplicó un cuadro de elaboración personal, el cual es un horario de una semana completa, donde el estudiantado marcó con una "X" las horas del día en que tenía tiempo libre. 


\section{Procedimiento}

Se aplicó los cuestionarios, el cuadro de tiempo libre y la toma de medidas kinantropométricas personalmente en las aulas de la institución. El investigador en conjunto con un grupo de colaboradores daba las indicaciones necesarias para completar los cuestionarios y el cuadro de tiempo libre e indicaba que, mientras las personas llenaban sus cuestionarios, se iba a ir llamando uno a uno para la toma de peso y talla, donde debían quitarse zapatos y medias además de objetos metálicos que pudieran afectar la medición del peso, ya que la báscula cuenta con detectores de impedancia magnética y los objetos metálicos pueden afectar la medición; una vez aclarado el procedimiento se iniciaba con la evaluación. En la toma de talla y peso se utilizaban los protocolos internacionales para tal fin.

Una vez finalizado el proceso anterior, se sistematizó la información recolectada y se realizó el respectivo análisis.

\section{Análisis estadístico}

Se realizó el análisis estadístico descriptivo para determinar promedios y desviaciones estándar
(DS) de las variables dependientes continuas y se calculará frecuencias absolutas y relativas en los casos de variables dependientes nominales. Se aplicaron $t$-student en grupos independientes para analizar las variables continuas con respecto al sexo. Además, se utilizó una Chi-Cuadrada para analizar las variables nominales Los análisis se realizarán con el paquete estadístico para las ciencias sociales (PASW 15.0 para Windows).

\section{Resultados}

\section{Cantidad de tiempo libre en una semana en tiempo lectivo}

En este apartado se describe las horas de tiempo libre que tienen los sujetos en una semana, para luego mostrar algunas de las actividades en las que las aprovechan los estudiantes. Para ello, se aplicó un análisis factorial de varianza de dos vías comparando la variable cantidad de tiempo de horas libres por semana según sexo y la permanencia en la universidad (un año, de dos a cuatro años) de un total de 121 (53 hombres y 68 mujeres) estudiantes (19.79 \pm 2.46 años), el cual se aprecia en la tabla 1 a continuación.

Tabla 1. Comparación de las variables cantidad de tiempo de horas libres por semana según sexo y la permanencia en la universidad (un año, de 2 a 4 años)

\begin{tabular}{|c|c|c|}
\hline Fuente de Varianza verdadera & $\mathrm{F}$ & Sig. \\
\hline Sexo & 3.42 & .067 \\
\hline Permanencia & 4.38 & $.038^{\star}$ \\
\hline Sexo-Permanencia & 7.46 & $.007^{\star \star}$ \\
\hline
\end{tabular}


La tabla anterior muestra que existe diferencias en la cantidad de tiempo libre (TL) total de lunes a domingo entre hombres y mujeres y hay diferencia entre la cantidad de TL de lunes a domingo según los años de permanencia. Por tanto el tiempo libre varía entre hombres y mujeres y entre semestres de permanencia en la universidad pero no hay interacción entre variables. Esto se observa en las figuras 1 y 2 a continuación.

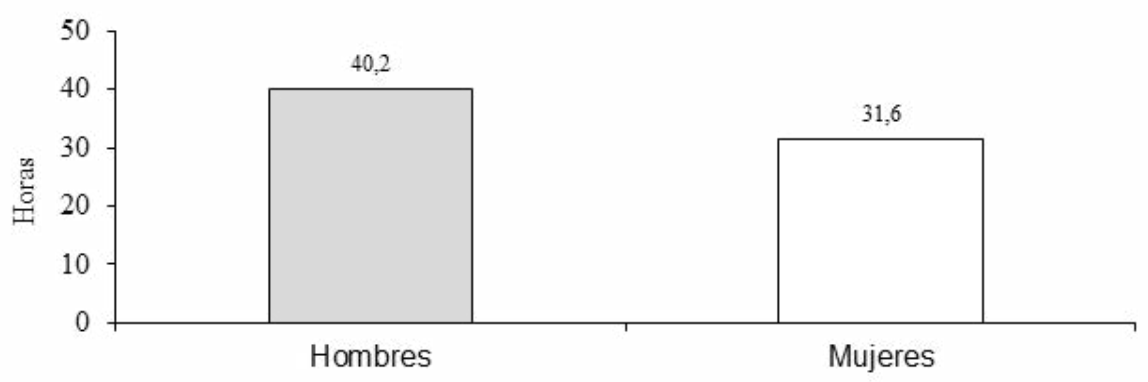

Figura 1 Promedio total de horas de TL por semana para hombres y mujeres a lo largo de sus estudios.

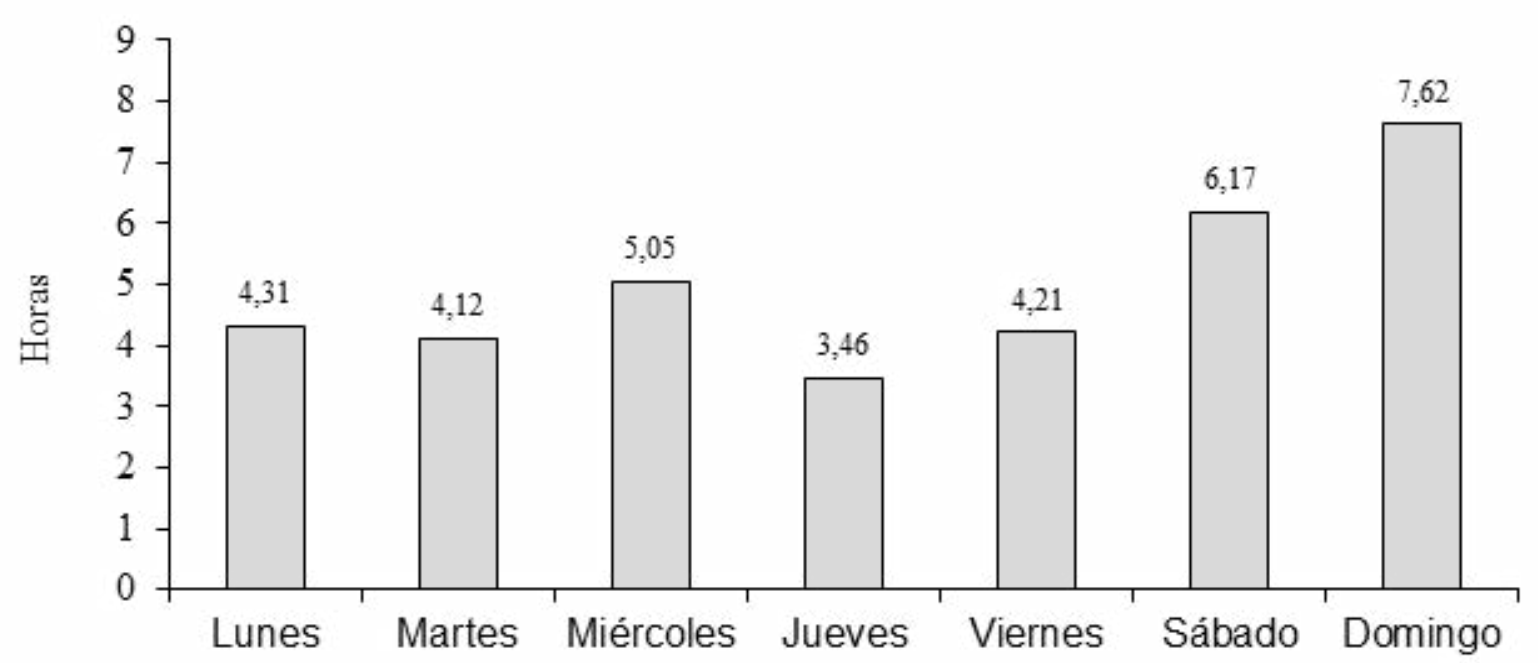

Figura 2. Promedio total de horas de TL por día de la semana para ambos sexos y años de permanencia en la Universidad.

El análisis Post-hoc de Bonferroni mostró que los días sábados y domingos, en comparación con los demás días de la semana, es cuando se presenta una mayor cantidad de horas de tiempo libre, y en los demás destaca el día miércoles.

Se pudo observar que tanto hombres y mujeres tienen un porcentaje importante de horas de tiempo libre mientras cursan sus estudios. Esto podrá refutar algunos de los resultados encontrados en los siguientes apartados a la hora de ponerlo de excusa para no hacer actividad física o deporte.

\section{Tipo de actividades en tiempo libre}

Seguidamente algunos ítems del cuestionario de hábitos de los jóvenes, que caracteriza los estilos de vida de los estudiantes universitarios y los deja en evidencia como estudiantes con hábitos sedentarios:

- En la relación sexo y el ítem "Durante la semana, si tiene un rato libre, ¿qué te gustaría hacer?" Según Chi-cuadrado de Pearson se obtuvo una $\chi^{2}=11.962^{\mathrm{a}}, \mathrm{p}=.008(\mathrm{p}<.05)$, por tanto, existe relación significativa, la actividad 
más frecuente mencionada fue intento hacer algo de deporte (45\% de la muestra total, $26.1 \%$ hombres y 18.9 mujeres). Seguida de navegar por internet $(44.1 \%$ de la muestra total, $19.8 \%$ de los hombres y $24.3 \%$ de las mujeres). Se aprecian diferencias según el sexo, que relacionadas con quienes intentan hacer algo de deporte como actividad en TL durante la semana, la mayor cantidad de personas en este caso son los hombres (26.1\%) mientras que en navegar por internet o jugar con la computadora el porcentaje de quien más las practica es de mujeres (24.3\%). De las otras opciones como "me voy de compras" y "me voy con los amigos a tomar cervezas" apenas obtuvieron un $7.2 \%$ y un $3.6 \%$ del total de la muestra, respectivamente. (Nota: Se presenta el estadístico de razón de verosimilitudes debido a que se superaba en más de un $25 \%$ las celdas con una frecuencia esperada inferior a 5).

- En otro ítem a ambos sexos a la pregunta “QQué tipo de impedimento encuentras?”, esto en relación con el porqué no realizan algún deporte, según Chi-cuadrado de Pearson se obtuvo una $\chi^{2}=1.038^{a}(p=792)$, no existe relación significativa; importante señalar que los estudiantes indican como mayor impedimento la falta de tiempo $(73.1 \%$ del total de la muestra, hombres $34.6 \%$, mujeres $38.5 \%$ ) entre otros como no tener a nadie con quien practicar deporte y problemas de salud (Nota: Se presenta el estadístico de razón de verosimilitudes debido a que se superaba en más de un $25 \%$ las celdas con una frecuencia esperada inferior a 5).
- Por otra parte, "navegar por internet" es la segunda actividad más realizada por los estudiantes. Se encontró una relación significativa entre sexo y el ítem “¿Cuánto tiempo dedicas diariamente al internet, entre semana?"; según Chi-cuadrado de Pearson se obtuvo una $\chi^{2}=8.057, \mathrm{p}=.045(\mathrm{p}<.05)$; a la pregunta la opción con mayor porcentaje fue "más de 120 minutos" (39.7\%), los hombres fueron mayoría(56.3\%), en el caso de las mujeres el porcentaje más alto se encontró en la casilla "entre 30 y 120 minutos" un 22.3\%. En la misma relación pero para el fin de semana, los universitarios dan como respuesta con mayor porcentaje "entre 30 y 120 minutos" (38.8\%), los hombres obtuvieron $19.0 \%$ y las mujeres 19.8\%, no hubo significancia, según Chi-cuadrado de Pearson se obtuvo una $\chi^{2}=$ $4.231(\mathrm{p}=.239)$.

Los estudiantes intentan hacer algo de deporte y navegar por internet como actividades diarias y exponen como impedimento para hacer deporte la falta de tiempo; las actividades mencionadas son de carácter sedentaro en los jóvenes universitarios, y se contradicen cuando indican que no tienen tiempo para realizar deporte, lo que responde el apartado siguiente.

\section{Nivel de actividad física}

Los resultados de este apartado se presentan según un parámetro. Se muestran los datos según los valores correspondientes a distintos niveles de actividad física establecidos por $\mathrm{FAO} / \mathrm{WHO} / \mathrm{UNU}$ (FAO, 2004).

Tabla 2. Clasificación de los estilos de vida según el nivel de actividad física

\begin{tabular}{lc}
\multicolumn{1}{c}{ Categoría } & Valores PAL (nivel de actividad física) \\
Estilo de vida sedentario o de actividad ligera. & $1.40-1.69$ \\
Estilo de vida activo o moderadamente activo. & $1.70-1.99$ \\
Estilo de vida de actividad vigorosa. & $2.00-2.40$ \\
\hline & Fuente: FAO/WHO/UNU (2001).
\end{tabular}


Según Chi-cuadrado de Pearson se obtuvo una $\chi^{2}=13.888 \mathrm{a}(\mathrm{p}<.05)$, existe una relación significativa entre sexo y la clasificación de nivel de actividad física en el periodo lectivo. Indistintamente del sexo durante el periodo lectivo, la mayor parte de los sujetos son sedentarios o muy poco activos; un $74.8 \%$ del estudiantado presentó esa condición (el $31.4 \%$ son hombres y el $43.4 \%$ son mujeres; es decir que, indistintamente del sexo, la mayor parte del estudiantado está en esta clasificación); los que tienen una actividad moderada y son activos son apenas un $13.2 \%$ de la población y los que son de actividad vigorosa o sea muy activos apenas un $14.9 \%$ esto durante el tiempo lectivo. Entonces el que no haya relación con el sexo quiere decir que, independientemente de que sean hombres o mujeres, los resultados son los mismos, por tanto no se puede decir que las mujeres sean menos activas o viceversa.

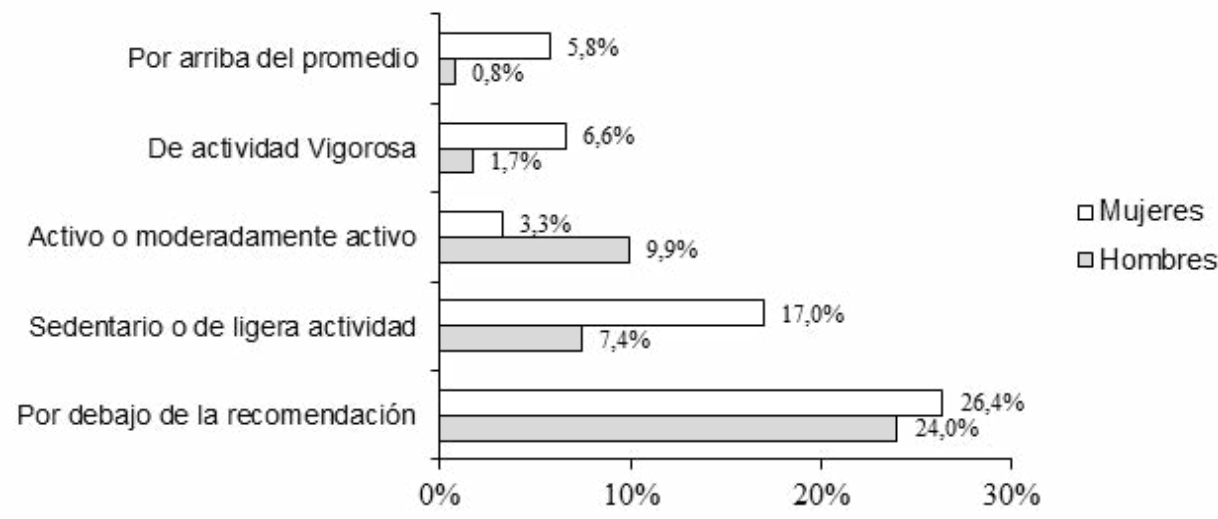

Figura 3. Distribución de estudiantes hombre y mujeres por nivel de actividad física en periodo de clases.

Según Chi-cuadrado de Pearson se obtuvo una $\chi 2=8.772 \mathrm{a}(\mathrm{p}<.05)$, no existe relación significativa entre sexo y la clasificación de nivel de actividad física en el periodo de vacaciones. Indistintamente del sexo, durante el periodo de vacaciones la mayor parte de los sujetos son sedentarios o muy poco activos; un $91.8 \%$ del estudiantado presentó esa condición (43.0\% son hombres y $48.8 \%$ mujeres; es decir que, indistintamente del sexo, la mayor parte del estudiantado está por debajo de la recomendación); los que tienen una actividad moderada o activos son apenas un $4.9 \%$ de la población y los que de actividad vigorosa o muy activos son apenas un $3.4 \%$, esto durante el tiempo de vacaciones. Entonces el que no haya relación con el sexo quiere decir los resultados son semejantes, por tanto no se puede decir que las mujeres sean menos activas o viceversa.

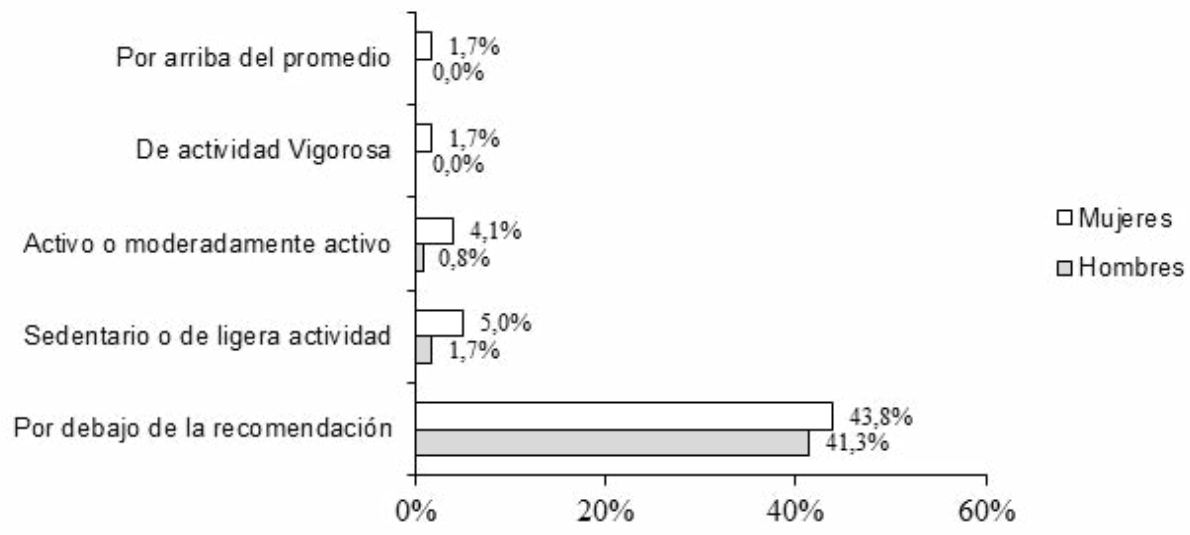

Figura 4. Distribución de estudiantes hombres y mujeres por nivel de actividad física en periodo de vacaciones. 
Importante destacar que son aún más sedentarios o pocos activos los estudiantes, indistintamente del sexo, en el periodo de vacaciones que el periodo de clases. Al conocer las características anteriores de los estudiantes universitarios, las cuales ratifican que son sedentarios en su mayoría y que a pesar de tener tiempo libre no realizan actividad física ni deporte, se dio un dato curioso en la siguiente variable.

\section{Prevalencia de obesidad}

La clasificación internacional para determinar el IMC para adultos con la que se trabaja es la que propone la Organización Mundial de la Salud: Bajo peso (<18.50), Normal (18.50 - 24.99), Sobrepeso (25.00-29.99), Obeso tipo I (30.00 - 34-99), Obeso tipo II (35.00 - 39.99) y Obesidad tipo III ( $\geq 40.00)$ (OMS, 1995).

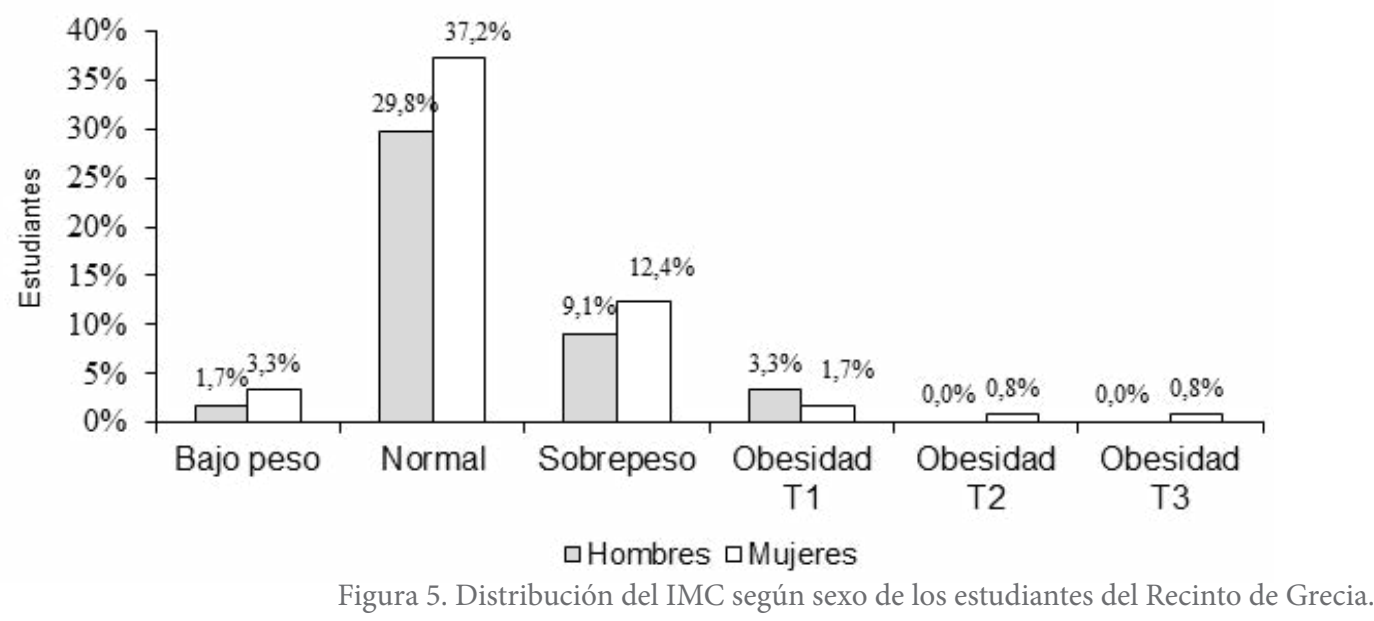

Con respecto a la relación entre sexo e índice de masa corporal (IMC), del total de la muestra, un $67 \%$ se encuentra en la clasificación " 18.5 24.99 peso adecuado" indistintamente del sexo, seguida de un $21.5 \%$ en la clasificación de " 25 29.99 sobrepeso". Según Chi-cuadrado de Pearson $\chi 2=3.877 \mathrm{a}(\mathrm{p}>.05)$, al no encontrarse relación significativa entre la clasificación del IMC y el sexo, la tendencia general mencionada antes es la misma para cada sexo.

\section{Discusión}

El tiempo libre tenido por los estudiantes universitarios en promedio de horas por día para hombres es de 5.74 y para las mujeres de 4.51 horas. Estos datos son consistentes con el tiempo libre reportado en otro análisis en estudiantes universitarios, conformado por una muestra de 494 estudiantes de las carreras del área de la salud del centro de estudios superiores Santo Tomás de Talca (Enfermería, Kinesiología, Técnico de Enfermería,
Técnico Laboratorista Dental), el cual reportó horas de TL para hombres de 6,10 y para las mujeres de 5,15 horas en promedio por día, lo que demuestra que los hombres disponen de más tiempo libre (Lemp y Behn, 2008). Por tanto, si poseen tiempo libre de horas al día, conocer a qué dedican el tiempo permite identificar prioridades, hábitos, actitudes e intereses en su vida cotidiana.

El tiempo libre, en el caso de la muestra, lo dedican, independientemente del sexo, a la siguientes opciones: intento hacer algo de deporte, me voy de compras, navegar por la Internet o juego con la computadora y me voy con los amigos a tomar cervezas; a las actividades como intentar hacer deporte y navegar por la Internet o jugar con la computadora en ese orden respectivamente coinciden en la opción de navegar por la internet con los resultados de Rangel y otros (2011), no así intentar hacer algo de deporte. Solamente en el uso de internet los estudiantes abarcan un tiempo de dos o más horas diarias de las seis o cinco horas de TL 
reportado por día por los estudiantes. Resultados concordantes con Ruiz y otros (2001), en cuyo estudio se muestra que los estudiantes dedican el tiempo libre a realizar actividades de ocio pasivo, entre ellas, ver televisión y manejar la computadora; Fernández (2005) encuentra que prefieren como actividades navegar por internet $y$, finalmente, el estudio de Lemp y Behn (2008) muestra la preferencia por ver televisión y salir con amigos. No se encontró estudios similares en el país.

Lo anterior responde a lo que a continuación se encontró en relación con el nivel de actividad física. Los datos encontrados según los valores correspondientes a distintos niveles de actividad física establecidos por $\mathrm{FAO} / \mathrm{WHO} / \mathrm{UNU}$ (FAO, 2004) revelan que existen bajos niveles de actividad física muy alarmantes, indistintamente del sexo y del periodo: lectivo o de vacaciones; la mayor parte de los sujetos son sedentarios o de actividad ligera, en ciclo lectivo un $71.9 \%$ presenta esta condición (el $43.7 \%$ son hombres y el $56.3 \%$ son mujeres) y el $50.4 \%$ está por debajo de la recomendación, y en periodo de vacaciones un $91.4 \%$ presenta esta condición (46.8\% son hombres y $53.2 \%$ mujeres) y el $85.1 \%$ está por debajo de la recomendación; es decir que, indistintamente del sexo, la mayor parte del estudiantado es sedentario o de actividad física ligera; los que tienen una actividad moderada o activos son apenas un $13.2 \%$ en ciclo lectivo y un $5 \%$ en periodo de vacaciones, y los que son de actividad vigorosa o muy activos un $14.9 \%$ en ciclo lectivo y $3.4 \%$ en periodo de vacaciones. Según su permanencia, no cambia la tendencia a ser sedentarios o pocos activos en ambos periodos.

A partir del análisis (nivel de actividad física y cumplimiento con las recomendaciones mínimas), los resultados correspondientes a hombres y mujeres presentan la misma tendencia, lo cual muestra altos niveles de sedentarismo y bajos niveles de cumplimiento de las recomendaciones, en los dos periodos estudiados (mayor aún en vacaciones). Pueden verse ciertas diferencias por sexo: las mujeres evidencian un sedentarismo o poca actividad física más elevada que los hombres.

Los resultados del estudio realizado por Barrantes (2010), en estudiantes asmáticos y no asmáticos del Instituto Tecnológico de Costa Rica (ITCR) en el año 2008, evidencian la misma tendencia de sedentarismo o actividad ligera; de la muestra estudiada por Barrantes, el $87 \%$ presenta patrones de actividad física que implican estilos de vida de tipo sedentario o de actividad ligera (un 75\% de ellos con niveles extremos de sedentarismo). Cabe señalar que la inactividad física no solo contribuye a las enfermedades crónicas no transmisibles (ECNT), sino que también puede predisponer a enfermedades mentales, la acumulación de estrés y un menor rendimiento escolar e incluso tener un efecto negativo en la interacción social (OPS, 2006).

En relación con la prevalencia de obesidad de los estudiantes el estudio, que a pesar de conocer lo sedentario de los estudiantes, del total de la muestra, un $66.9 \%$ se encuentra en la clasificación “18.5-24.99 peso adecuado" indistintamente del sexo, seguida de un $21.5 \%$ en la clasificación de " 25 29.99 sobrepeso". Y por sexo la tendencia general mencionada antes es la misma para cada sexo. Los hombres presentaron un $67.9 \%$ para peso adecuado y un $20.8 \%$ en sobrepeso y las mujeres un $66.2 \%$ en peso adecuado y un $22.1 \%$ en sobrepeso. Para las clasificaciones de obesidad 1, 2 y 3 los casos van disminuyendo aunque sí se presentan datos de sujetos en estas clasificaciones (Hombres 7.5\%, 0\% y $0 \%$ respectivamente; Mujeres 2.9\%, $1.5 \%$ y $1.5 \%$ respectivamente).

Fue ligeramente similar el estudio realizado por Barrantes (2010), con una población estudiantil del (ITCR), Sede Cartago, con 1358 estudiantes, con edades entre 16 y 28 años de grado (programas de Bachillerato y Licenciatura), reporta que durante el 2008 en esta institución el IMC fue para los hombres $1 \%$ de bajo peso, $75 \%$ de peso adecuado, $19 \%$ de sobrepeso y un $5 \%$ de obesidad 1, y las mujeres $1.6 \%$ de bajo peso, $83.6 \%$ de peso adecuado, $13.1 \%$ de sobrepeso y $1.6 \%$ obesidad 1 .

El IMC de ambos estudios refleja una consistencia en todos sus rangos, donde la población universitaria en estudio y la del ITCR el mayor porcentaje de sujetos se encuentra en un índice de peso adecuado, seguido de sobrepeso. Importante recalcar que en sobrepeso y obesidad 1 en ambos 
estudios los hombres son los que presentan mayor porcentaje en relación con las mujeres. Al comparar estos datos con la población en el nivel nacional, la Encuesta Nacional de Nutrición 2008-2009 para Costa Rica mostró una prevalencia de un $33,2 \%$ de sobrepeso y un $26,5 \%$ de obesidad en mujeres de 20 a 44 años, y en hombres de 20 a 64 años el sobrepeso es de $43,5 \%$ y un 18,9\% de obesidad (Ministerio de Salud y Ministerio de Deporte y Recreación, 2011), los datos en el nivel nacional se encuentran muy por encima de los datos reportados por los universitarios en relación con el sobrepeso y obesidad.

\section{Conclusiones}

Los estilos de vida de la población estudiantil son caracterizados por tener 40.23 y 31.57 horas promedio de tiempo libre en una semana para hombres y mujeres respectivamente; similar o mayor al que poseen estudiantes en otras universidades y se ve disminuido por el tiempo de permanencia en la universidad. Las actividades realizadas por hombres y mujeres en tiempo libre durante una semana principalmente son las siguientes: intento hacer algo de deporte y navegar por Internet, y con menos interés ir de compras y salir a tomar cervezas con los amigos.

El nivel de actividad física de los estudiantes es caracterizado por el sedentarismo en ambos periodos estudiados: clases y de vacaciones; pues no cumplen con las recomendaciones mínimas para la práctica de actividad física que permita mantener un estado de salud adecuado y prevenir la aparición de otras enfermedades crónicas, así como la mortalidad prematura. El estilo de vida de los estudiantes se vuelve más sedentario durante el periodo de vacaciones en comparación con el periodo de clases. Eso evidencia que la universidad aunque no solventa las necesidades requeridas, si repercute en la condición de sedentarismo de los estudiantes en cierta medida.

La prevalencia de obesidad de los estudiantes fue baja en comparación con la población nacional e internacional. La mayoría de los universitarios (tanto hombres como mujeres) reporta índices de masa corporal dentro del rango normal; sin embargo, los estilos de vida sedentarios y el tiempo de permanencia en la universidad representan un riesgo importante para el aumento de estos valores en niveles de sobrepeso u obesidad. En el caso de quienes ya presentan estas condiciones, el riesgo se asocia con el mantenimiento o agudización, con el consecuente incremento del riesgo relacionado con enfermedades crónicas y mortalidad prematura. 


\section{Referencias}

ACSM, (2010). ACSM's Guidelines for Exercise Testing and Prescription. 8a. ed. Philadelphia: Lippincott Williams \& Wilkins.

Barbosa, N., Sánchez, C., Vera, J., Pérez, W., Thalabard, J-C y Rieu, M. (2007). "A Physical Activity Questionnaire: Reproducibility and Validity". Journal of Sport Science and Medicine, 6, 505-518. Recuperado de http:// www.jssm.org/vol6/n4/18/v6n4-18pdf.pdf, el 2 de noviembre de 2014 .

Brango, J. y Villanueva, F. (s.f.). "Estilos de vida saludables". Revista EDU-FISICA. Grupo de Investigación Edufisica. ISSN 2027- 453X. Recuperado de http://www.edu-fisica.com/ Revista\%205/ESTILOS-VS.pdf, el 20 de mayo del 2014.

Barrantes, K. (2010). Prevalencia de asma y caracterización de estudiantes asmáticos del ITCR, Sede Cartago. Aportes para una propuesta de intervención. Tesis Universidad Nacional de Costa Rica, Campus Presbítero Benjamín Núñez, Heredia, Costa Rica.

FAO (2004). Human energy requirements. Report of a Joint FAO/WHO/UNU ExpertConsultation no. 1. Rome: FAO. Recuperado en: ftp://ftp. fao.org/docrep/fao/007/y5686e/y5686e00.pdf, el 10 de setiembre del 2014.

Fernández, A. (2005). ¿A qué dedican el tiempo libre los españoles? Recuperado de: http:// www.facilisimo.com/encuesta/, el día 25 de octubre del 2013.

Gil, P. et ál (2011). Cuestionario Hábitos de los estudiantes. Enviado por correo electrónico directamente del autor el día 9 de junio del 2014.

Gómez, J.R. Jurado, M. I. Viana, B. H. Da Silva, M. E. Hernández, A. (2005). "Estilos y calidad de vida”. Revista Digital - Buenos Aires - Año 10 -
$\mathrm{N}^{\circ}$ 90. Recuperado de http://www.efdeportes. com/efd90/estilos.htm, el 11 de octubre del 2014.

JAMA, (2003). "Obesidad". The Journal of the American Medical Association. Vol 289, No. 14. Recuperado de http://jama.ama-assn.org/ content/suppl/2003/04/11/289.14.1880.DC1/ pdfpat040903.pdf, el 23 de junio del 2014.

Oblitas, L. (2006). Psicología de la salud y calidad de vida. Segunda edición. International Thomson Editores, S.A. México.

Olivares, S. Lera, L. y Bustos, N. (2008). “Etapas del cambio, beneficios y barreras en actividad física y consumo de frutas y verduras en estudiantes universitarios de Santiago de Chile". Revista Chile Nutrición. 35(1); 25-35. Recuperado de: http://www.scielo.cl/scielo.php?pid=S0717$75182008000100004 \&$ script $=$ sci_arttext; el 25 de junio del 2014.

OMS (1995). Physical status: the use of and interpretation of anthropometry, report of a WHO expert committee. Editor Geneva: World Health Organization. Recuperado de: http://www.who.int/iris/handle/10665/37003; el 24 de junio del 2014.

OMS, (2011). Obesidad. Organización Mundial de la Salud. Recuperado de http://www.who.int/ topics/obesity/es/, el 15 de abril del 2014

Osorio, M. (2002). "Experiencia docente en la formación de estudiantes en el área de salud ocupacional, Caracas, Venezuela”. Revista Cubana de salud y Trabajo 3(1-2) pp. 65-67. Recuperado de http://bvs.sld.cu/revistas/rst/ vol3_\%201-2_02/rst11102.pdf, el 14 de junio del 2014.

Prieto, A. (2003). "Modelo de promoción de salud con énfasis en Actividad Física para una Comunidad Estudiantil Universitaria”. Rev. Salud Pública, 5 (3), 284-300. Recuperado de: http://www.scielosp.org/pdf/rsap/ v5n3/20030.pdf, el día 20 de marzo del 2014. 
Rangel, N. Ochoa, M. (2011). "El tiempo libre de los estudiantes universitarios de la Facultad de Comercio y Administración de Tampico". Revista Ciencias UAT. Vol. 23; No. 1. Recuperado de: http://intranet.uat.edu.mx/ cienciauat/Lists/noticiasciencia2/DispFormx. aspx?ID=275, el día 20 de junio del 2014.

Rodríguez, J. (1995). Promoción de la salud y prevención de la enfermedad: estilos de vida y salud, en Psicología social de la Salud. Madrid. Ed. Síntesis.

Ruiz , F. García, M. y Hernández, A. (2001). "Disponibilidad de tiempo libre del alumnado almeriense de enseñanza secundaria postobligatoria y de segundo ciclo de la Universidad de Almería, y tipos de actividades con que lo ocupan". Efdeportes Revista Digital Buenos Aires 7(34). Recuperado de: http:// www.efdeportes.com/efd34b/tlibre1.htm, el día 23 de setiembre del 2014.

Sanabria, P. González, L. Urrego, D. (2007). “Estilo de vida saludable en profesionales de la salud colombianos, Bogotá, Colombia”. Revista Med 15(002) pp.207-217 Recuperado de http:// redalyc.uaemex.mx/pdf/910/91015208.pdf, el 23 de junio del 2014.

Scott, B., Morrow, J., Jackson, A. y Dunn, A. (2000). "Variables related to meeting the CDC/ ACSM physical activity guidelines". Medicine and Scienc in Sports and Exercise, 32(12), 2087-2092. Recuperado de http://journals. lww.com/acsmmsse/Abstract/2000/12000/ Variables_related_to_meeting_the_CDC_ ACSM_physical.19.aspx, el 29 de abril del 2014.

Seignón, C. (2008). Estilos de vida y nutrición en universitarios de la Benemérita Universidad Autónoma de Puebla (BUAP). Recuperado de http://www.archivos.ujat.mx/dip/ divulgacion $\% 20 \mathrm{y} \% 20$ video $\% 20$ cinetifico $\% 20$ 2008/DACS/ELaraM.pdf, el 10 de abril del 2014. 
